\title{
Togitare
}

ARTIGO ORIGINAL

\section{FATORES ASSOCIADOS À PROPOSIÇÃO DO DIAGNÓSTICO DE ENFERMAGEM: ATRASO NO CRESCIMENTO EM ADOLESCENTES}

\author{
Kadyjina Lúcio Daiane Batista ${ }^{1}$ \\ Karolayne Cabral Matias ${ }^{1}$ (1) \\ Ricaelly de Medeiros Cavalcanti ${ }^{1}$ (i) \\ Renata Marinho Fernandes ${ }^{1}$ (1) \\ Marcos Venícius de Oliveira Lopes ${ }^{2}$ (1) \\ Ana Luisa Brandão de Carvalho Lira ${ }^{1}$ (1)
}

\section{RESUMO}

Objetivo: analisar os fatores socioeconômicos e clínicos associados à proposição do diagnóstico de enfermagem atraso no crescimento em adolescentes.

Método: estudo transversal em escolas públicas de uma cidade do Nordeste do Brasil. Amostra com 385 adolescentes entre 10 e 19 anos. A coleta ocorreu de junho a setembro de 2017. A análise de dados ocorreu no IBM SPSS statistic.

Resultados: os fatores que apresentaram associação com as características definidoras "Estatura final inferior ao alvo", "Maturação sexual retardada", "Diminuição da massa óssea", "Velocidade de crescimento abaixo do esperado" e "Diminuição da massa óssea" foram: sexo, anos de estudo, estado civil e massa muscular. Os fatores relacionados "Efeitos indesejáveis de terapias" e "Privação emocional" associaram-se à idade e ao sexo, respectivamente.

Conclusão: Esses fatores podem influenciar direta ou indiretamente na proposição diagnóstica, contribuindo na acurada detecção do problema, oportunizando ações precisas e resultados positivos em saúde.

DESCRITORES: Crescimento; Desenvolvimento do Adolescente; Adolescente; Diagnóstico de Enfermagem; Processo de Enfermagem.

\section{FACTORES ASOCIADOS CON LA PROPOSICIÓN DEL DIAGNÓSTICO DE ENFERMERÍA: RETRASO EN EL CRECIMIENTO DE LOS ADOLESCENTES}

\section{RESUMEN}

Objetivo: analizar los factores socioeconómicos y clínicos asociados con la proposición del diagnóstico de enfermería retraso del crecimiento en adolescentes. Método: estudio transversal en escuelas públicas de una ciudad del Nordeste de Brasil. Muestra con 385 adolescentes de entre 10 y 19 años. La recolección tuvo lugar de junio a septiembre de 2017. Los datos obtenidos fueron analizados con la ayuda de IBM SPSS statistic. Resultados: Los factores que se asociaron a las características definitorias "Estatura final por debajo del objetivo", "Retraso en la maturación sexual", "Disminución de la masa ósea", "Velocidad de crecimiento por debajo del esperado" y "Disminución de la masa ósea" fueron: sexo, años de estudio, estado civil y masa muscular. Los factores relacionados con los "Efectos indeseables de las terapias" y la "Privación emocional" se asociaron con la edad y al sexo, respectivamente. Conclusión: Estos factores pueden influir directa o indirectamente en la propuesta diagnóstica, contribuyendo a la detección precisa del problema y proporcionando acciones precisas y resultados positivos para la salud.

DESCRIPTORES: Crecimiento; Desarrollo del Adolescente; Adolescente; Diagnóstico de Enfermería; Proceso de Enfermería. 
O crescimento da estatura é um processo biológico, resultado de uma série de interações genéticas e ambientais, expresso pelo aumento do tamanho corporal. É um processo dinâmico da evolução, em função do tempo. O crescimento ocorre de forma não-linear e pode ser suscetível a influências externas, como o estado nutricional, aspectos ambientais e capacidade das cartilagens de responderem aos estímulos de crescimento ${ }^{(1-2)}$.

A fase da adolescência é marcada por um complexo processo de crescimento e desenvolvimento biopsicossocial. Segundo o Ministério da Saúde, a adolescência compreende a segunda década da vida (10 a 20 anos incompletos), sendo caracterizada por um intenso crescimento e desenvolvimento físico, fortemente influenciado pelo ambiente social, econômico e cultural(2).

É considerada uma fase de grande vulnerabilidade a fatores externos que podem influenciar e provocar danos à estatura do indivíduo, o que demonstra a importância do acompanhamento do crescimento para avaliação das condições de saúde do adolescente ${ }^{(3)}$. A suspeita clínica do atraso no crescimento serve de alerta para o profissional, que deve buscar revisão minuciosa da história familiar, de saúde e social do adolescente ${ }^{(4)}$.

Sabe-se que na adolescência ocorre um maior crescimento do esqueleto, denominado estirão puberal ${ }^{(3)}$. Entretanto, podem existir problemas nesse processo que resultem no atraso deste crescimento. Nesse contexto, salienta-se o papel do enfermeiro, como profissional instruído e respaldado legalmente para acompanhar e avaliar o adolescente nas diversas transformações que essa faixa etária proporciona ${ }^{(5)}$.

Nesse sentido, o cuidar na enfermagem exige decisões e intervenções fundamentais para a avaliação do estado de saúde do indivíduo. Torna-se também necessário que o enfermeiro, a partir do levantamento do estado de saúde do indivíduo sob seu cuidado, identifique os diagnósticos de enfermagem manifestados nessa clientela ${ }^{(6)}$.

Os diagnósticos de enfermagem (DE) representam um julgamento clínico sobre as respostas humanas às condições de saúde/processos de vida, ou vulnerabilidade, de indivíduos, famílias, grupos ou comunidades ${ }^{(7)}$. Entretanto, a NANDA-I não apresenta diagnóstico de enfermagem relacionado ao atraso no crescimento em seu sistema de classificação atual.

A NANDA-I, em sua versão 2012-2014, apresentava o diagnóstico de enfermagem 'Atraso no crescimento e no desenvolvimento no Adolescente' ${ }^{\prime(8)}$. No entanto, na edição de 2015-2017, esse DE foi excluído, por acreditar que investigações adicionais deveriam ser realizadas com a finalidade de distinguir os conceitos crescimento e desenvolvimento ${ }^{(9)}$.

Diante dessa problemática, autores ${ }^{(10-11)}$ desenvolveram estudos voltados à validação da proposição diagnóstica 'Atraso no Crescimento em Adolescentes Escolares', através das etapas de análise de conceito, conteúdo e validação clínica. Esse novo diagnóstico pode ajudar no direcionamento da assistência de enfermagem ao adolescente.

Desta forma, visando a contribuir para o avanço da enfermagem, o estudo teve como objetivo analisar os fatores socioeconômicos e clínicos associados à proposição do diagnóstico de enfermagem Atraso no crescimento em adolescentes. 
Trata-se de um estudo transversal, realizado em escolas estaduais de uma cidade do Nordeste do Brasil. A cidade possui 131 escolas estaduais, distribuídas em quatro zonas: Leste com 41 unidades; Sul com 33; Oeste com 31; e, Norte com 26. Essas escolas foram agrupadas por zonas em ordem numérica no Microsoft Excel, e a seleção ocorreu mediante sorteio em cada grupo, utilizando a função aleatorioentre. Essa ferramenta tem a capacidade de selecionar aleatoriamente números em um intervalo predefinido, os números selecionados equivaliam às escolas que entrariam na amostra. Foram selecionadas duas escolas em cada zona, totalizando oito instituições de ensino.

A população do estudo foi composta por alunos com idade entre 10 a 19 anos. Para a determinação do cálculo amostral, fez-se uso de uma constante pré-definida(12), 55, a qual foi multiplicada pelo número de características da proposição diagnóstica Atraso no crescimento em adolescentes, sete. Assim, o tamanho amostral foi de 385 adolescentes.

Como critérios de inclusão estipularam-se: idade entre 10 a 19 anos, estar regularmente matriculado em uma das escolas selecionadas e residir ou ter comunicação com os pais biológicos. Esse último critério foi lançado tendo em vista que a pesquisa necessita de dados concernentes aos pais biológicos. Os critérios de exclusão foram: estar em estado físico e/ou mental comprometido incapaz de responder aos questionamentos, estar de licença médica ou estar ausente da escola no momento da coleta dos dados.

Foi utilizado como instrumento de coleta de dados um formulário de entrevista e exame físico, construído a partir do resultado da análise de conceito realizada em estudo anterior $^{(10)}$. Esse contém dados sociodemográficos e clínicos, as características definidoras e fatores relacionados da proposição diagnóstica, bem como o referencial empírico para a mensuração da presença ou ausência das características definidoras.

As variáveis identificadas como características definidoras eram: baixa estatura por idade; baixo peso por idade; estatura final inferior ao alvo genético; maturação sexual retardada; velocidade de crescimento abaixo do esperado; e, diminuição da massa óssea. Atraso no surto de crescimento puberal, apesar de também ser uma característica da proposição diagnóstica, não foi avaliada pela necessidade de submeter os adolescentes a radiografia de mão e punho, que, além dos recursos onerosos, iria expor os indivíduos a radiação. Em relação às variáveis identificadas como fatores relacionados, têm-se: distúrbios genéticos; doenças crônicas; efeitos indesejáveis de terapias; estresse físico prolongado; e, privação emocional.

A coleta de dados ocorreu de julho e setembro de 2017, e foi realizada por dois pósgraduandos e três graduandos em enfermagem, devidamente treinados.

Para o exame físico, foi usada balança digital portátil com bioimpedância, da marca Beurer® (com precisão de 100 gramas). Na avaliação corporal, o adolescente subiu na balança descalço, sem adornos e posicionado no centro da plataforma. Essas informações foram importantes para mensurar as características definidoras baixo peso por idade e diminuição da massa óssea. Para estatura, foi utilizada uma fita métrica inelástica com os adolescentes descalços, em posição ortostática, braços ao longo do corpo, pés unidos, cabeça orientada no plano horizontal de Frankfurt, após inspiração e joelhos esticados ${ }^{(13)}$. Dados esses, utilizados como parâmetros para avaliação da presença das características: baixa estatura por idade; estatura final inferior ao alvo genético; e, velocidade do crescimento abaixo do esperado. Os dados encontrados foram comparados com gráficos da Organização Mundial de Saúde $(\mathrm{OMS})^{(13)}$, além de informações adquiridas na própria entrevista ao adolescente, como estatura média dos pais.

A proposta de Tanner foi utilizada como método para avaliação da característica maturação sexual retardada. Usou-se como base a observação das características secundárias, considerando o autorrelato de desenvolvimento mamário e dos pelos pubianos, no sexo feminino; e, o aspecto dos órgãos genitais e pelos pubianos masculinos ${ }^{(3,14)}$. Para a autoavaliação, foram utilizadas figuras autoexplicativas e instruído o adolescente sobre os estágios e as modificações concernentes a elas, por meio do auto-tanner. Posteriormente, 
os adolescentes marcavam no desenho em qual estágio se encontravam. Esse foi o meio adotado para evitar desconforto ou constrangimento por parte do entrevistado, visto que a pesquisa foi realizada no ambiente escolar.

Para os fatores relacionados distúrbios genéticos, doenças crônicas e efeitos indesejáveis de terapias, foi indagado ao aluno se possuía algumas dessas características através de uma lista de distúrbios genéticos, doenças permanentes e terapias que pudessem causar atraso no crescimento, conforme literatura ${ }^{(10-11)}$. Para o estresse físico prolongado, foi indagado sobre atividades que envolvessem esforço físico intenso, aos que realizam esse tipo de exposição foi verificado o nível de gordura corporal, mediante balança com bioimpedância. O fator relacionado foi considerado presente quando os padrões de gordura estivessem abaixo do padrão de normalidade de acordo com a idade e sexo(10-11).

Para o fator privação emocional, foi exposto diversas situações de privação afetiva e emocional que o adolescente pudesse ter vivenciado. Essas situações podem reduzir a liberação transitória de hormônio do crescimento e repercutir de maneira negativa no seu pico de crescimento ${ }^{(11)}$.

Utilizou-se do programa IBM SPSS Statistic versão 20.0 for Windows para a análise estatística descritiva dos dados sociodemográficos e clínicos, e para as frequências das características definidoras e fatores relacionados. Foram calculadas as frequências relativas e absolutas das variáveis categóricas, as medidas de tendência central e dispersão dos dados numéricos, como também constatada a normalidade dos dados, por meio do teste de Kolmogorov-Smirnov $(p<0,05)$.

O projeto foi aprovado pelo Comitê de Ética em Pesquisa da instituição responsável, com parecer favorável número 1.713.820.

\section{RESULTADOS}

A amostra apresentou uma predominância do sexo feminino, 218 (56,6\%), pardos 201 (52,2\%), vivendo sem companheiro 319 (82,9\%). Medianas de 12 anos de estudo, 14 anos de idade, renda familiar de dois salários mínimos e quatro integrantes na família. Concernente aos dados clínicos, a massa muscular apresentou-se com índice normal 299 (77,6\%), conforme apresentado na Tabela 1.

Tabela 1 - Caracterização sociodemográfica e clínica dos adolescentes de escolas estaduais. Natal, RN, Brasil, 2017 (continua)

\begin{tabular}{llll} 
Variáveis & & $\mathbf{n}$ & \% \\
\hline \multirow{2}{*}{ Sexo } & Feminino & 218 & 56,6 \\
\cline { 2 - 4 } & Masculino & 167 & 43,3 \\
\hline \multirow{2}{*}{ Estado civil } & Sem companheiro & 319 & 82,9 \\
\cline { 2 - 4 } & Com companheiro & 66 & 17,1 \\
\hline \multirow{2}{*}{ Raça } & Parda & 201 & 52,2 \\
\cline { 2 - 4 } & Branca & 82 & 21,3 \\
\cline { 2 - 4 } & Negra & 75 & 19,5 \\
\hline
\end{tabular}




\begin{tabular}{|c|c|c|c|c|c|c|}
\hline & Amarela & & 27 & & 7 & \\
\hline \multirow{3}{*}{ Massa muscular } & Normal & & 299 & & 77,6 & \\
\hline & Baixo & & 79 & & 20,5 & \\
\hline & Alto & & 7 & & 1,8 & \\
\hline Variáveis & Média & Mediana & DP & Mínimo & Máximo & Valor $p^{1}$ \\
\hline Idade* & 14,45 & 14 & 2,07 & 10 & 19 & 0 \\
\hline Anos de estudo & 12,28 & 12 & 2,04 & 9 & 16 & 0 \\
\hline Integrantes ${ }^{\S}$ & 4,4 & 4 & 1,68 & 2 & 18 & 0 \\
\hline Renda Familiar" & 1,86 & 2 & 1,09 & 0 & 8 & 0 \\
\hline
\end{tabular}

Fonte: Autor (2020).

Legenda: ${ }^{1}$ Teste de Kolmogorov Smirnov; DP Desvio Padrão; *Idade em anos;§ Integrantes da família; ๆRenda familiar em salários mínimos (um salário mínimo equivalente a $\mathrm{R} \$ 937,00$ ).

Na Tabela 2, estão dispostas as variáveis sociodemográficas e clínicas e sua associação com características definidoras e fatores relacionados da proposição diagnóstica, Atraso no Crescimento em adolescentes escolares.

Tabela 2 - Variáveis sociodemográficas e clínicas e sua associação às características definidoras e fatores relacionados da proposição diagnóstica Atraso no crescimento em adolescentes escolares. Natal, RN, Brasil, 2017

\begin{tabular}{|c|c|c|c|c|c|c|c|c|}
\hline & Sexo & $\begin{array}{c}\text { Estado } \\
\text { civil }\end{array}$ & Raça & $\begin{array}{l}\text { Anos de } \\
\text { estudo }\end{array}$ & Idade & Integrantes & $\begin{array}{l}\text { Renda } \\
\text { familiar }\end{array}$ & $\begin{array}{c}\text { Massa } \\
\text { muscular }\end{array}$ \\
\hline CD1 & $0,474^{\star}$ & $0,274^{\star}$ & $0,825^{\star \star}$ & $0,931^{\pi}$ & $0,167 \pi$ & 0,889 १ & 0,9699 & $0,023^{\star \star \S}$ \\
\hline CD2 & $0,556^{\star *}$ & $0,441^{\star}$ & $0,593^{\star \star}$ & $0,149^{\uparrow}$ & $0,048^{\text {15 }}$ & $0,935^{\natural}$ & $0,505^{\natural}$ & $0,058^{\star *}$ \\
\hline CD3 & $0,000 * \star \S$ & $0,036^{\star * \S}$ & $0,689 * *$ & $0,000^{\text {ศร }}$ & 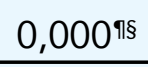 & $0,117^{\natural}$ & $0,874^{\pi}$ & $0,249 * *$ \\
\hline CD4 & $0,005^{\star \star \S}$ & $0,025^{\star \star \S}$ & $0,661^{* *}$ & $0,000^{\text {ศร }}$ & 0,000 १ร & $0,750^{\natural}$ & $0,873^{\pi}$ & $0,686^{\star *}$ \\
\hline CD5 & 0,260 ** & 0,460 ** & $0,643^{\star \star}$ & $0,083^{\pi}$ & $0,141^{\pi}$ & $0,972^{\pi}$ & 0,769 ฯ & $0,026^{\star \star \S}$ \\
\hline CD6 & 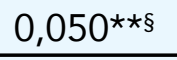 & 0,097 ** & $0,192^{\star \star}$ & 0,000 ๆร & 0,000 雨 & $0,857^{\natural}$ & $0,307^{\natural}$ & $0,000 * * \S$ \\
\hline FR1 & $0,566^{*}$ & $0,829 *$ & $0,821^{\star \star}$ & $0,636^{\pi}$ & $0,782^{\pi}$ & $0,882^{\natural}$ & $0,317^{\natural}$ & $0,866^{\star *}$ \\
\hline FR2 & $0,153^{\star \star}$ & $0,825^{\star \star}$ & $0,565^{\star \star}$ & $0,345^{\natural}$ & $0,563^{\pi}$ & $0,195^{\natural}$ & $0,086^{\natural}$ & $0,477^{\star \star}$ \\
\hline FR3 & $0,116^{*}$ & $0,534^{*}$ & 0,460 ** & $0,220^{\natural}$ & 0,020 १ร & $0,209^{\Uparrow}$ & $0,237^{\natural}$ & $0,228^{\star *}$ \\
\hline FR4 & $0,102^{\star}$ & $0,530 *$ & $0,657^{\star *}$ & $0,558^{\natural}$ & $0,440^{\pi}$ & $0,920^{\natural}$ & $0,599^{\natural}$ & $0,943^{\star *}$ \\
\hline FR5 & $0,001 * \star \S$ & $0,329 * *$ & $0,494^{\star \star}$ & $0,233^{\natural}$ & 0,702 & $0,724^{9}$ & $0,136^{\natural}$ & $0,371 * *$ \\
\hline
\end{tabular}

Fonte: Autor (2020).

Legenda: CD1: Baixa estatura por idade; CD2: Baixo peso por idade; CD3: Estatura final inferior ao alvo genético; CD4: Maturação sexual retardada; CD5: Velocidade de crescimento abaixo do esperado; CD6: Diminuição da massa óssea. FR1: Distúrbios genéticos; FR2:Doenças Crônicas; FR3: Efeitos indesejáveis de terapias; FR4: Estresse físico prolongado; FR5: Privação emocional. *Teste exato de Fisher, ${ }^{\star *}$ Teste de Qui-quadrado, ๆ Teste de U de Mann-Whitney,§ valor p: $\leq 0,05$. 
Em relação à associação entre os componentes da proposição diagnóstica Atraso no crescimento em adolescentes escolares e as variáveis sociodemográficas e clínicas, ressalta-se que a variável sexo associou-se com as características "Estatura final inferior ao alvo genético", "Maturação sexual retardada" e "Diminuição da massa óssea".

Salienta-se que para a característica Estatura final inferior ao alvo genético, dos $55(14,2 \%)$ indivíduos que apresentaram essa característica, 38 (9,8\%) foram do sexo masculino. Já maturação sexual retardada foi identificada em $124(32,2 \%)$ indivíduos, desses, $83(21,5 \%)$ foram do sexo feminino. E a diminuição da massa óssea apresentou um quantitativo de $62(16,1 \%)$ adolescentes do sexo feminino.

A variável estado civil se relacionou com "Estatura final inferior ao alvo genético" e "maturação sexual retardada", com predomínio de adolescentes sem companheiros nas duas situações.

No que se refere aos anos de estudo, foi observado que esse dado se associou a "Estatura final inferior ao alvo genético", "Maturação sexual retardada" e "Diminuição da massa óssea", assim como a variável idade. Todavia, essa teve associação ainda com a característica "Baixo peso por idade".

A variável massa muscular associou-se com a característica "Baixa estatura por idade", "Velocidade de crescimento abaixo do esperado" e "Diminuição da massa óssea". Com relação à análise dos fatores relacionados, nota-se que a variável sexo associou-se com o fator relacionado "Privação emocional". Já a idade associou-se com "Efeitos indesejáveis de terapias".

\section{DISCUSSÃO}

A monitorização do crescimento do adolescente é consensualmente aceita como essencial para instrumento de avaliação e controle do aspecto nutricional(15). No estudo, a baixa estatura associou-se significativamente com o índice de massa muscular dos adolescentes. Corroborando, artigo afirma que o pico de velocidade do crescimento em estatura é diretamente proporcional ao índice de massa muscular magra, estatura, conteúdo mineral ósseo e maturação sexual|(16).

Verificou-se que o baixo peso em adolescente associou-se com a idade. Isso ocorre devido às características da própria idade, como: maior demanda de nutrientes para o crescimento, maturação de órgãos, reservas de energia e dependência familiar; as crianças e os adolescentes são vulneráveis à desnutrição(17).

Autores demonstram que, em ambos os sexos, as médias de peso e altura tiveram aumentos gradativos com avanço da idade e estágios de maturação(18). Esse dado corrobora com a presente pesquisa, cujo fator relacionado desnutrição crônica não esteve presente na população estudada, entretanto reconhece a influência que essa causa tem no atraso do crescimento. Houve uma baixa prevalência de baixo peso em adolescentes, com média de idade de 14 anos.

A característica Estatura inferior ao alvo genético esteve presente na amostra e associou-se com as características sociais anos de estudo e idade. Assim, quando realizado o cálculo para o alvo genético, muitos adolescentes ainda não tinham chegado à sua estatura final aplicando-se, principalmente, ao sexo masculino. Destaca-se que essa característica pode ser facilmente observada pelo enfermeiro na atenção primária à saúde, por não depender de recursos onerosos.

A maturação sexual retardada foi a característica definidora que apresentou maior 
prevalência, e associou-se ao sexo. A literatura aborda sua ocorrência em aproximadamente 3\% da população, sendo mais frequente no sexo masculino. Nas mulheres, essa característica geralmente é secundária a uma doença subjacente ${ }^{(19)}$. Entretanto, no presente estudo a maturação sexual foi mais prevalente nas mulheres. Dos adolescentes que apresentaram essa característica, $67 \%$ eram do sexo feminino. Todavia, deve-se salientar que os participantes realizaram uma autoavaliação, podendo ter ocorrido erros no julgamento dos seus estágios.

Quanto à variável estado civil em associação com essa característica, percebeu-se que adolescentes que iniciam a vida sexual mais cedo passam por modificações corporais também mais precocemente ${ }^{(20)}$. Essa característica associou-se também com idade e anos de estudo. Autores demonstram que a idade se mostra importante tanto na análise da antropometria como na análise puberal de adolescentes ${ }^{(19)}$.

A característica velocidade de crescimento abaixo do esperado esteve presente em $5,7 \%$ dos adolescentes. A velocidade de crescimento pode ser analisada em fases distintas, esse é o principal critério de normalidade, visto que esse é um processo dinâmico ${ }^{(21)}$. Destarte, essa é uma característica importante de ser avaliada pelos profissionais, visto que analisa uma série temporal e não uma medida isolada de tempo. A velocidade de crescimento no menino ocorre em torno dos 14 anos, já na menina tende a acontecer mais cedo, geralmente ocorrendo em torno de 12 anos $^{(22)}$.

A velocidade de crescimento associou-se com o índice de massa muscular. Estudo demonstra que o crescimento máximo ocorre durante o estágio 4 de Tanner e nesse período também existe a formação extensiva de massa muscular ${ }^{(23)}$.

As variáveis idade e anos de estudo apresentaram associação com Diminuição da massa óssea. A variável idade mostra-se importante tanto na análise da antropometria, como na análise puberal de adolescentes ${ }^{(24)}$. Nessa faixa etária, tanto o sexo feminino quanto o masculino já poderiam ser analisados quanto ao atraso, pois para os dois grupos as características sexuais secundárias já deveriam estar presentes ${ }^{(19)}$.

A característica Diminuição da massa óssea esteve presente em 24,7\% amostra. Percebeu-se que adolescentes mais novos apresentavam índice de massa óssea inferior, quando comparado aos mais velhos, a mesma proporção aconteceu com os anos de estudo. Uma pesquisa com adolescentes do sexo feminino constatou menores valores de massa óssea nas participantes com 10 anos de idade e valores superiores em meninas de 17 anos ou mais. Em geral, essas possuem maior risco de acometimentos ósseos como a osteoporose $^{(25)}$.

Esse fato corrobora com os achados do presente estudo, em que a diminuição da massa óssea esteve associada com a variável sexo e massa muscular. Assim, a avaliação óssea reflete a maturidade esquelética e pode ser útil na análise de distúrbios do crescimento. Os atrasos significativos na idade óssea podem ser encontrados na puberdade tardia ou uma doença crônica subjacente, enquanto que condições de crescimento rápido, como a puberdade precoce, podem resultar em um avanço da idade óssea ${ }^{(22,25)}$.

Nota-se que a variável sexo também se associou com o fator Privação emocional. Não há estudos que façam associação específica do fator relacionado à variável sexo. A privação emocional é o gerenciamento de emoções negativas que causam privação sentimental e consequentemente pode causar deficiência transitória da libertação do hormônio do crescimento. Dependendo do momento e da intensidade na vida desse adolescente em que a privação se inseriu, poderão se desenvolver diferentes respostas ${ }^{(26)}$.

A privação emocional pode ser ocasionada por algum fator que faça o adolescente querer regressar a um período anterior à privação, onde houve uma perda de algo que foi bom em determinado momento e foi, então, retirado por certo período. $O$ atraso no crescimento é encontrado em grande parte de famílias que estão dentro do contexto de separação, cuidados à saúde inadequados ou alguma discórdia. Como ferramenta para 
ajudar na conciliação das relações, está o diálogo, estabelecendo sentimentos de confiança e afeto no cuidado, para minimização da situação de privação emocional|(27).

Já a idade associou-se com o fator relacionado Efeitos indesejáveis de terapias. Estudos apontam que tratamentos como radioterapias, quimioterapia, ou que usam de corticoides e antirretrovirais causam considerável atraso no crescimento da estatura, de órgãos ou puberal e consequentemente podem alterar o metabolismo ósseo e a composição corporal|(28).

Em todos os tratamentos citados anteriormente, quando ocorridos em anos iniciais da adolescência, há probabilidade de causar efeitos indesejáveis no crescimento. Nota-se que essas terapias ecoam negativamente no crescimento do adolescente, principalmente quando acontecem em conjunto com importantes modificações corporais, nas quais o organismo precisaria de energia para o processo de crescimento ${ }^{(28)}$.

Dessa forma, observa-se que o conhecimento de fatores socioeconômicos e clínicos, quando associados ao reconhecimento de fatores etiológicos e características expressados nessa clientela, podem auxiliar no rastreamento precoce do atraso do crescimento em adolescentes. O estudo em questão associa essas variáveis, buscando aumentar e facilitar o entendimento sobre o fenômeno do crescimento, bem como demonstrar quais fatores podem repercutir no seu processo natural.

Para a enfermagem, o estudo disponibiliza uma ferramenta útil para avaliação de variáveis nas consultas ao adolescente, principalmente por não dispor de recursos onerosos para a avaliação, além de contribuir para o estado da arte na profissão por apresentar uma nova proposta diagnóstica coadunada à análise clínica e de fatores sociodemográficos. Para a sociedade, além de apresentar uma forma de monitorização do crescimento dos adolescentes, viabiliza instrumentos e procedimentos em saúde que possam promover progressos na qualidade da assistência a essa clientela, além de melhores prognósticos para a idade adulta.

Como limitação do estudo, ressalta a temporalidade, uma vez que, para analisar o fenômeno do crescimento, orientam-se abordagens longitudinais maiores que seis meses. Assim, sugere-se a aplicação do produto em estudos longitudinais e com diferentes populações adolescentes.

\section{CONCLUSÃO}

Conclui-se que os fatores socioeconômicos e clínicos podem influenciar direta ou indiretamente os componentes da proposição do diagnóstico de enfermagem "Atraso no crescimento em adolescentes escolares".

As variáveis sexo e anos de estudo associaram-se às características definidoras "Estatura final inferior ao alvo", "Maturação sexual retardada" e "Diminuição da massa óssea". A variável estado civil teve associação com as características definidoras "Estatura final inferior ao alvo" e "Maturação sexual retardada". A variável massa muscular associouse as características definidoras "Velocidade de crescimento abaixo do esperado" e "Diminuição da massa óssea". Quanto aos fatores relacionados, destaca-se que "Efeitos indesejáveis de terapias" se associou à variável idade e "Privação emocional" associou-se à variável sexo.

\section{AGRADECIMENTOS}


Esse estudo contou com a subvenção da Coordenação de Aperfeiçoamento de Pessoal de Nível Superior (CAPES), por meio de bolsa de estudos (cód. 011).

\section{REFERÊNCIAS}

1. Damiani D, Damiani D, Steinmetz L. Déficit de crescimento: como abordar e tratar? Pediatr. mod [Internet]. 2014 [acesso em 10 jun 2019]; 50(1). Disponível em: http://pesquisa.bvsalud.org/portal/ resource/pt/lil-712196?lang=es.

2. Ministério da Saúde (BR). Proteger e cuidar da saúde de adolescentes na Atenção Básica. Brasília: Ministério da Saúde, 2017.

3. ChulanI VL, Gordon LP. Adolescent Growth and Development. Prim Care [Internet]. 2014 [acesso em 10 jun 2019]; 41(3). Disponível em: https://doi.org/10.1016/j.pop.2014.05.002.

4. Rogol AD, Hayden GF. Etiologies and early diagnosis of short stature and growth failure in children and adolescents. J Pediatr. [Internet]. 2014 [acesso em 08 jul 2019]; 164(5). Disponível em: https://doi. org/10.1016/j.jpeds.2014.02.027.

5. Silva AB da, Oliveira JL, Magalhães JM, Sales MCV. A assistência do enfermeiro da atenção básica ao adolescente com dependência química. Revista Interdisciplinar [Internet]. 2014 [acesso em 08 jul 2019]; 7(4). Disponível em: https://revistainterdisciplinar.uninovafapi.edu.br/index.php/revinter/article/view/526.

6. Silva PEL da, Monma JMA, Souza ACO, Barbosa EV, Carvalho FA, Lemos PEB, et al. O adolescente com a síndrome da imunodeficiência adquirida: a utilização da NANDA e da SAE. Rev Recien [Internet]. 2017 [acesso em 08 jul 2019]; 7(21). Disponível em: https://doi.org/10.24276/ rrecien2358-3088.2017.7.21.49-55.

7. Herdman TH, Kamitsuru S. Diagnósticos de enfermagem da NANDA-I: definições e classificação 20182020. Porto Alegre: Artmed; 2018.

8. Hedman TH. NANDA International nursing diagnoses: definitions and classification. New Jersey: WileyBlackwell; 2012.

9. Hedman TH, Kamitsuru S. Diagnóstico de enfermagem da NANDA: definições e classificações 20152017. [NANDA Internacional]. Porto Alegre: Artmed; 2015.

10. Andriola IC. Construção do diagnóstico de enfermagem atraso do crescimento no contexto do adolescente [dissertação]. Natal (RN): Centro de Ciências da Saúde, Universidade Federal do Rio Grande do Norte; 2016.

11. Lúcio KDB. Proposição diagnóstica para o atraso no crescimento em adolescentes escolares: análise de conteúdo e validação clínica [dissertação]. Natal (RN): Centro de Ciências da Saúde, Universidade Federal do Rio Grande do Norte; 2017.

12. Swanson SA, Lindenberg K, Bauer S, Crosby RD. A Monte Carlo investigation of factors influencing latent class analysis: an application to eating disorder research. International J Eat Disord [Internet]. 2012 [acesso em 08 jul 2019]; 45(5). Disponível em: https://doi.org/10.1002/eat.20958.

13. World Health Organization (WHO). Physical status: the study and interpretation of anthropometry. Genebra: WHO; 1995.

14. Tanner JM. Growth at adolescence.2 ed. Oxford: Blackwell Scientific Publications; 1962.

15. Tambalis KD, Panagiotakos DB, Arnaoutis G, Psarra G, Maraki M, Mourtakos S, et al. Establishing cross-sectional curves for height, weight, body mass index and waist circumference for 4- to 18-year-old 
Greek children, using the Lambda Mu and Sigma (LMS) statistical method. Hippokratia. [Internet]. 2015 [acesso em 08 jul 2019]; 19(3). Disponível em: https://www.ncbi.nlm.nih.gov/pmc/articles/PMC4938472/.

16. Oliveira PM de, Silva FA da, Oliveira RMS, Mendes LL, Pereira Netto M, Cândido APC. Associação entre índice de massa de gordura e índice de massa livre de gordura e risco cardiovascular em adolescentes. Rev. Paul. Pediatr. [Internet]. 2016 [acesso em 08 jul 2019]; 34(1). Disponível em: http:// www.scielo.br/scielo.php?pid=S0103-05822016000100030\&script=sci_arttext\&tIng=pt.

17. Silva C de S, Cabral PC, Galvão SF, Diniz AS, Arruda IKG de, Santos CM dos. Estado nutricional de crianças e adolescentes admitidos para internação em um hospital universitário. Rev. bras. pesqui. saúde [Internet]. 2016 [acesso em 08 jul 2019]; 17(2). Disponível em: https://periodicos.ufes.br/rbps/article/ view/13186.

18. Godoi Filho JR de M, Farias E dos S. Aptidão física de escolares do sudoeste da Amazônia Ocidental em diferentes estágios de maturação sexual. Rev. bras. educ. fís. esporte [Internet]. 2015 [acesso em 08 jul 2019]; 29(4). Disponível em: http://dx.doi.org/10.1590/1807-55092015000400631.

19. Wolfenden H, Ryan F. Delayed puberty. Paediatrics and child health [Internet]. 2014 [acesso em 08 jul 2019]; 24(3). Disponível em: https://doi.org/10.1016/j.paed.2013.09.006.

20. Spinola MCR, Béria JU, Schermann LB. Fatores associados à iniciação sexual em mães de 14 a 16 anos em Porto Alegre / RS, Brasil. Ciênc. saúde coletiva. [Internet]. 2017 [acesso em 08 jul 2019]; 22(11). Disponível em: http://dx.doi.org/10.1590/1413-812320172211.00082016.

21. Frittoli RB, Longhi BS, Silva AM, Barros Filho A de A, Monteiro MAR de G, Appenzeller S. Effects of the use of growth hormone in children and adolescentes with juvenile idiopathic arthritis: a systematic review. Rev. Bras. Reumatol. [Internet]. 2017 [acesso em 08 jul 2019]; 57(2). Disponível em: http://dx.doi. org/10.1016/j.rbre.2016.07.009.

22. Amin N, Mushtaq T, Alvi S. Fifteen-minute consultation: The child with short stature. Arch Dis Child Educ Pract Ed [Internet]. 2015 [acesso em 13 jul 2019] 100(4). Disponível em: http://dx.doi.org/10.1136/ arch dischild-2014-306488.

23. Cairo RC de A, Silva LR, Bustani NC, Marques CDF. Iron deficiency anemia in adolescents: a literature review. Nutr. Hosp. [Internet]. 2014 [acesso em 13 jul 2019]; 29(6). Disponível em: http://dx.doi. org/10.3305/nh.2014.29.6.7245.

24. Medeiros RMV, Arrais RF, Azevedo JCV de, Rêgo JTP do, Medeiros JA de, Andrade RD de, et al. Contribuição das características antropométricas na predição dos estádios de maturação puberal de jovens do sexo masculino. Rev. paul. pediatr. [Internet]. 2014 [acesso em 13 jul 2019]; 32(3). Disponível em: http://dx.doi.org/10.1590/0103-0582201432313.

25. Fortes CMT, Goldberg TBL, Kurokawa CS, Silva CC, Moretto MR, Biason TP, et al. Relationship between chronological and bone ages and pubertal stage of breasts with bone biomarkers and bone mineral density in adolescents. J. Pediatr. [Internet]. 2014 [acesso em 13 jul 2019]; 90(6). Disponível em: http://dx.doi.org/10.1016/j.jped.2014.04.008.

26. Silva CYG da, Milani RG. Adolescência e tendência antissocial: o Rap como expressão de uma privação emocional. Psicol. cienc. prof. [Internet]. 2015 [acesso em 13 jul 2019]; 35(2). Disponível em: https://doi.org/10.1590/1982-370301572013.

27. Simões C, Santos AC, Canha L, Matos MG de. Resiliência na adolescência: género e a idade fazem a diferença? J. Clin. Child Adolesc. [Internet]. 2019 [acesso em 13 jul 2019]; 10(1). Disponível em: http:// revistas.lis.ulusiada.pt/index.php/rpca/article/view/2627.

28. Molinari PCC, Lederman HM, Lee ML de M, Caran EMM. Assessment of the late effects on bones and on body composition of children and adolescents treated for acute lyphocytic leukemia according to Brazilian protocols. Rev paul. pediatr. [Internet]. 2017 [acesso em 13 jul 2019]; 35(1). Disponível em: https://doi.org/10.1590/1984-0462/;2017;35;1;00005. 
COMO REFERENCIAR ESTE ARTIGO:

Batista KLD, Matias KC, Cavalcanti RM, Fernandes RM, Lopes MV de O, Lira ALB de C. Fatores associados à proposição do diagnóstico de enfermagem: atraso no crescimento em adolescentes. Cogitare enferm. [Internet]. 2021 [acesso em "colocar data de acesso, dia, mês abreviado e ano"]; 26. Disponível em: http://dx.doi. org/10.5380/ce.v26i0.69988.

*Artigo extraído da dissertação de mestrado "Proposição diagnóstica para o atraso no crescimento em adolescentes escolares: análise de conteúdo e validação clínica". Universidade Federal do Rio Grande do Norte, 2017.

Recebido em: 31/10/2019

Aprovado em: 03/08/2020

Editora associada: Tatiane Herreira Trigueiro

Autor Correspondente:

Kadyjina Lúcio Daiane Batista

Universidade Federal do Rio Grande do Norte - Natal, RN, Brasil

E-mail:kadyjinadaiane@gmail.com

Contribuição dos autores:

Contribuições substanciais para a concepção ou desenho do estudo; ou a aquisição, análise ou interpretação de dados do estudo - KLDB, KCM, RMC, RMF, MVOL, ALBCL

Elaboração e revisão crítica do conteúdo intelectual do estudo - KLDB, MVOL, ALBCL

Aprovação da versão final do estudo a ser publicado - KLDB, KCM, RMC, RMF, MVOL, ALBCL

Responsável por todos os aspectos do estudo, assegurando as questões de precisão ou integridade de qualquer parte do estudo - KLDB, ALBCL

Copyright () 2021 Este é um artigo em acesso aberto distribuído nos termos da Licença Creative Commons Atribuição, que permite o uso irrestrito, a distribuição e reprodução em qualquer meio desde que o artigo original seja devidamente citado. 